\title{
Cavity Hydration and Competitive Binding in Methylated $\beta$-Cyclodextrin
}

\author{
Denilson M. de Oliveira and Dor Ben-Amotz ${ }^{*}$ \\ Department of Chemistry, Purdue University, West Lafayette, IN 47907, USA. \\ *E-mail: bendor@purdue.edu
}

\section{Supplementary Information}

Experimental Details. Aqueous solutions of benzene-d6 (Sigma-Aldrich) and heptakis(2,6-diO-methyl)- $\beta$-cyclodextrin (Sigma-Aldrich) were prepared with ultrapure filtered water (Milli-Q UF Plus, 18.2 $\mathrm{M} \Omega \mathrm{cm}$, Millipore). Raman spectra were obtained at $20^{\circ} \mathrm{C}$ using an Ar-ion 514.5 $\mathrm{nm}$ laser with $\sim 20 \mathrm{~mW}$ of power at the sample and 5 minutes of integration time. The backscattered Raman photons were collected from the sample using a $20 \times$ long working distance microscope objective ( $\mathrm{NA}=0.42$, Mitutoyo Inc.) and transmitted with an optical fiber to a 300 mm imaging spectrograph (SpectraPro300i, Acton Research Inc.) equipped with a $300 \mathrm{gr} / \mathrm{mm}$ grating and a CCD camera (Princeton Instruments Inc. Pixis 400B).

The SMCR decomposition is performed using measured Raman spectra of pure water and aqueous solutions containing the host and/or guest molecules, collected under identical conditions. It is particularly important to assure that the temperatures of the two samples are the same (to within at least $0.1^{\circ} \mathrm{C}$ ), and wavelength changes resulting from barometric pressure variations are compensated for by including a helium calibration lamp line in each measured Raman spectrum (as in our previous Raman-MCR studies). In practice, obtaining properly converged SC spectra requires accurately establishing the background underlying the SC peaks of interest (using a linear or quadratic fit to the local background of the SC spectrum). 
TLS Spectral Decomposition. Let $X$ be the spectrum of a solution of some mixture of host and/or guest molecules, represented as a $1 \times n$ vector (where $n$ is the number of frequency values in the spectrum). If the spectra of the free and bound species are known, then total least squares (TLS) can be used to decompose $X$ into free and bound components. Specifically, let $P$ be a $2 \times n$ matrix of pure components (free and bound) spectra and $b$ is a $1 \times 2$ vector that contains the (as yet unknown) spectral weights of the two components in $X$, such that

$$
X=b P
$$

The coefficients in $b$ may be determined using TLS as follows

$$
b=X P^{T}\left(P P^{T}\right)^{-1}
$$

where $P^{T}$ represents the transpose of matrix $P$. Note that if the spectra $X$ and the two component spectra in $P$ are all normalized to unit area (over the frequency range of the band of interest), then the elements of the vector $b$ are the spectral weights of the free and bound components.

Determination of Benzene Binding Constant and Raman Cross Section Change. A total least squares (TLS) fit of the C-D band obtained from a mixture of benzene-d6 and Me- $\beta$-CD to a linear combination of the free and bound benzene-d6 C-D bands can be used to retrieve the relative areas of free and bound benzene-d6 in the mixture. More specifically, when this fit is performed with all the C-D bands normalized to unit area, then the results yield the relative spectral weight of the free and bound components in the mixture C-D band. Converting these spectral weights to the corresponding component concentrations requires knowing the relative Raman cross sections of the free and bound benzene-d6 C-D stretch band. In the present case, the free benzene cross section is implicitly known (e.g., from measuring a saturated solution of benzene), and thus the concentration of free benzene-d6 in the mixture can be determined from 
the TLS fit results. If the mixtures used to perform these fits are all constrained to have a total benzene-d6 concentration of $10 \mathrm{mM}$, then mass balance (combined with the measured spectrum of a saturated aqueous benzene solution) can be used to obtain the concentration of bound benzene, as follows

$$
\begin{aligned}
& c_{\text {free }}=f_{\text {free }}\left(\frac{A_{\text {mix }}}{A_{\text {sat }}}\right) c_{\text {sat }} \\
& c_{\text {bound }}=c_{\text {total }}-c_{\text {free }}
\end{aligned}
$$

where $A_{\text {sat }}$ and $c_{\text {sat }}$ are the area and concentration $(22.9 \mathrm{mM})$ of a saturated solution of benzene, respectively; $A_{\text {mix }}$ is the area of a solution with known total benzene concentration, and $f_{\text {free }}$ is the TLS fraction (spectral weight) of the free benzene in the mixture. Thus, the ratio of the C-D stretch Raman cross sections of the bound and free benzene may be obtained as follows (where subscripts indicate the area and concentration of free and bound benzene obtained as explained above):

$$
\frac{\sigma_{\text {bound }}}{\sigma_{\text {free }}}=\frac{\left(\frac{A_{\text {bound }}}{c_{\text {bound }}}\right)}{\left(\frac{A_{\text {free }}}{c_{\text {free }}}\right)} \approx 1.4 \pm 0.1
$$

The above considerations may also be used to quantify the host-guest binding equilibrium constant. The formation of a 1:1 host/guest complex is usually represented by a complexation reaction of the form

$$
H+G \rightleftharpoons H G
$$

with an equilibrium constant given by

$$
K=\frac{[H G]}{[H][G]}
$$


Thus, the measured fraction of bound benzene-d6 (guest) along with the total concentrations benzene-d6 and Me- $\beta-\mathrm{CD}$ (host) may be used to obtain $K$, as illustrated in Figure 2(B). Alternatively, given the Raman cross section ratio in Eq. 6, one may determine $K$ from measurements of aqueous solutions in equilibrium with a liquid benzene-d6 phase. In this case the free benzene concentration is pinned to its saturated concentration and the measured C-D band of the aqueous solution can be used to obtain the concentration of bound benzene-d6 and the ratio of the complexed and free host concentrations.

Notice that the above equations do not include the water molecules in the free host which are displaced upon binding the guest. This is equivalent to assuming that the concentration of pure water is expressed in mole fraction units. In order to directly consider competitive binding of water and benzene it is more convenient to use the same units for all concentrations, and thus express the above equilibrium and equilibrium constant as follows:

$$
\begin{gathered}
K^{\prime}=\frac{[H G]\left[\mathrm{H}_{2} \mathrm{O}\right]^{n}}{\left[{\left.\mathrm{H}\left(\mathrm{H}_{2} \mathrm{O}\right)_{n}\right][G]}^{(G]}\right.} \\
H\left(\mathrm{H}_{2} \mathrm{O}\right)_{n}+G \rightleftharpoons H G+n \mathrm{H}_{2} \mathrm{O}
\end{gathered}
$$

Note that $K^{\prime}=K\left[\mathrm{H}_{2} \mathrm{O}\right]^{n}$ where $n$ is the number of water molecules bound in the cavity of the free host, and thus $K^{\prime}$ and $K$ have substantially different numerical values. For the complexation of benzene with Me- $\beta$-CD our results indicate that $K \sim 101(1 / \mathrm{M})$ and thus $K^{\prime}=K_{1}\left[H_{2} O\right]^{n}$ $\left(M^{n-1}\right)$, is estimated to be $5 \times 10^{10} \leq K^{\prime} \leq 3 \times 10^{12}$, since $5 \leq n \leq 6$ is the average number of cavity-bound water molecules in the free host, as determined from our Raman-MCR measurements (obtained as further described in the next sub-section). Thus, the equilibrium constant $K^{\prime}$ provides a direct measure of the competitive binding of water and benzene in the host cavity, as Eq. 8 indicates that the ratio of benzene-complexed to water-filled host cavities 
would be equal to $K^{\prime}$ if the concentrations of unbound water and benzene were both (hypothetically) scaled to a value of $1 \mathrm{M}$.

Determination of the Number of Water Molecules in the Me- $\beta$-CD Cavity. The number of cavity-bound water molecules $(n)$ in Me- $\beta-C D$ was evaluated using Eq. 10:

$$
n=\frac{55.4}{[H G]} \frac{A_{w}-A_{b}}{A_{w a t e r}}
$$

where $[H G]$ is estimated using the binding constant $K$ above; $55.4(\mathrm{M})$ is the concentration of pure water at $20^{\circ} \mathrm{C}$; and $A_{w}, A_{b}$ and $A_{\text {water }}$ represent the areas under the $\mathrm{OH}$ stretch band for the water-filled Me- $\beta-\mathrm{CD}$, benzene-filled Me- $\beta-\mathrm{CD}$, and pure water (as depicted in Fig. 1c of the main manuscript). The $A_{w}$ area was scaled to reflect the measured concentration of the benzenefilled Me- $\beta-C D$. Thus, given that our results indicate that $92 \leq K \leq 110$, Eq. 10 implies that $5 \leq n \leq 6$. Note that this estimated range of the number of cavity-bound water molecules assumes that there is no binding-induced change in the Raman cross section of water. 